\title{
Liquid biopsy as an option for predictive testing and prognosis in patients with lung cancer
}

\author{
Alvida Qvick ${ }^{1,2^{*}}$ D, Bianca Stenmark ${ }^{1,2}$, Jessica Carlsson ${ }^{3}$, Johan Isaksson ${ }^{4,5,6}$, Christina Karlsson ${ }^{7}$ and \\ Gisela Helenius ${ }^{1,2}$
}

\begin{abstract}
Background: The aim of this study was to investigate the clinical value of liquid biopsy as a primary source for variant analysis in lung cancer. In addition, we sought to characterize liquid biopsy variants and to correlate mutational load to clinical data.

Methods: Circulating cell-free DNA was extracted from plasma from patients with lung cancer $(n=60)$ and controls with benign lung disease $(n=16)$. Variant analysis was performed using the AVENIO ctDNA Surveillance kit and the results were correlated to clinical and variant analysis data from tumor tissue or cytology retrieved from clinical routine diagnostics.
\end{abstract}

Results: There were significantly more variants detected in lung cancer cases compared to controls $(p=0.011)$, but no difference between the histological subgroups of lung cancer was found $(p=0.465)$. Furthermore, significantly more variants were detected in patients with stage IIIb-IV disease compared to patients with stage I-IIla (median 7 vs $4, p=0.017)$. Plasma cfDNA mutational load was significantly associated with overall survival $(p=0.010)$. The association persisted when adjusted for stage and ECOG performance status (HR: 3.64, 95\% Cl 1.37-9.67, $p=0.009$ ). Agreement between tumor and plasma samples significantly differed with stage; patients with stage IIIb-IV disease showed agreement in $88.2 \%$ of the cases with clinically relevant variants, compared to zero cases in stage I-IIla $(p=0.004)$. Furthermore, one variant in EGFR, two in KRAS, and one in BRAF were detected in plasma but not in tumor samples.

Conclusion: This study concludes that in the vast majority of advanced NSCLC patients a reliable variant analysis can be performed using liquid biopsy from plasma. Furthermore, we found that the number of variants in plasma is associated with prognosis, possibly indicating a strategy for closer follow up on this crucial patient group.

\section{Introduction}

Lately, targeted therapy and immune checkpoint inhibition have revolutionized treatment for a subset of patients with non-small cell lung cancer (NSCLC) (Tsao et al. 2016). So far, drug targets are almost exclusively found in adenocarcinomas (AC), which account for about

\footnotetext{
*Correspondence: alvida.qvick@regionorebrolan.se

1 Dept. of Laboratory Medicine, Örebro University Hospital, Södra Grev. Roseng., 70185 Örebro, Sweden

Full list of author information is available at the end of the article
}

$40 \%$ of all lung cancer cases (Tsao et al. 2016). At present, there are approved drugs for lung cancer, targeting tumor cells with mutated EGFR, ALK and ROS1 fusions, and mutated BRAF (Tsao et al. 2016). Additionally, immune checkpoint inhibition that activates the immune system to target the tumor, such as monoclonal antibodies against PD1 and PD-L1, has further advanced the treatment of lung cancer (Brahmer et al. 2015).

The emergence of targeted therapies has dramatically increased the need for comprehensive tumor genotyping to predict optimal treatment as well as monitoring 
of the response. In turn, increased understanding of the genomic landscape of tumors has resulted in several basket trials investigating tumor agnostic treatments, with some drugs already receiving approval by the Food and Drug Administration in the US. This includes the antiPD-1 immune checkpoint inhibitor pembrolizumab and the NTRK fusion-targeting larotrectinib (Patnaik et al. 2015; Le et al. 2015; Drilon et al. 2018). The indication for treatment with pembrolizumab in the agnostic setting includes a mismatch repair deficient tumor resulting in an unstable genome with a high tumor mutational burden (TMB) (https://www.fda.gov/drugs/ drug-approvals-and-databases/fda-approves-pembrolizu mab-adults-and-children-tmb-h-solid-tumors). However, an agnostic approach still requires tumor material, which is especially challenging in lung cancer considering the sparse tissue material obtained in many cases. Difficulties in tissue sampling is due to tumor localization and comorbidities that normally prevent surgical biopsies, and at times even core needle biopsies, and diagnosis is often based on forceps biopsies obtained by bronchoscopy or fine needle aspirates. An alternative source of tumor material could be the use of circulating biomarkers obtained for example through a blood sample.

Circulating cell-free DNA (cfDNA) is a major part of the term liquid biopsy and has been extensively studied across several cancer types with promising results (Lin et al. 2017; Gao et al. 2017; Jiang et al. 2016; Spindler et al. 2017; Zhou et al. 2016). The proportion of cfDNA that originates from the tumor, called circulating tumor DNA (ctDNA), could be used to detect diagnostic, predictive, and prognostic biomarkers (Schwarzenbach et al. 2011; Stroun et al. 1989; Nie et al. 2015; Rolfo et al. 2014). Currently, ctDNA is already in use clinically for variant detection in EGFR as an option for routine follow-up of resistance mutations in patients with NSCLC (Wu et al. 2018). This is based on the use of ultra-sensitive assays, such as droplet digital PCR, for specific variants. To obtain information about a larger spectrum of genetic alterations, the method of choice is currently next generation sequencing (NGS). This method has been employed in other studies to analyze EGFR mutations in ctDNA from patients with NSCLC (Marchetti et al. 2015; Douillard et al. 2014). However, the performance of the tests was variable, between 50 and $80 \%$ sensitivity, compared to tissue or cytology samples, and in need of further improvement for ctDNA to be established as a clinical biomarker. Sequencing of larger genomic regions, to calculate the TMB for the clinical application of response to immunotherapy, has also been performed in NSCLC on plasma (Koeppel et al. 2017; Gandara et al. 2018; Andrew et al. 2017). The studies reported some scattered results regarding the TMB agreement with tumor tissue which might indicate that the sensitivity is again at the core of the problem.

In the present study, liquid biopsy was evaluated as a primary source for clinical variant analysis using a large NGS panel of 197 genes on plasma samples from patients with lung cancer and a benign lung disease control group. Detection of targetable variants as well as agreement in variants between plasma cfDNA and tumor samples were evaluated. In addition, the total number of variants, used as a simplified version of TMB, was associated to clinical parameters.

\section{Materials and methods \\ Study participants}

Patients referred to the lung clinic at Örebro University Hospital with suspicion of lung cancer were invited to participate in the study. Samples were collected during routine investigation, prior to diagnosis. Inclusion criteria consisted of: (1) patient with suspicion of lung cancer and (2) age above 18 years. Exclusion criteria included: (1) cancer of other origin than lung, (2) sample not collected prior to diagnosis, (3) inadequate tumor material for diagnosis and (4) insufficient cfDNA quantity for analysis. This resulted in a final cohort of 61 study participants with lung cancer and 16 with benign lung diseases including different types of inflammation, fibrosis and noduli, in and around the lung tissue. The latter group was consequently used as a control group, and is hereafter referred to as such. Survival status was retrieved from medical journals (followed until 5th of February 2020) and at time of retrieval 33 of 60 cancer patients were deceased with a median follow up time of 11.0 months (Additional file 1: Table S1). The study was approved by the regional ethics committee board in Uppsala (Dnr 2015-400) and participants gave written informed consent before inclusion.

\section{Sample collection and extraction}

Blood was collected in Cell-Free DNA ${ }^{\mathrm{TM}}$ BCT tubes (Streck, Omaha, NE) and plasma was retrieved by a two-step centrifugation; $2000 \times g$ for $10 \mathrm{~min}$ followed by $16,000 \times g$ for $10 \mathrm{~min}$. Plasma was stored at $-80^{\circ} \mathrm{C}$ until extraction of cfDNA was performed on $4 \mathrm{~mL}$ plasma, using the QIAsymphony DSP Circulating DNA kit on the QIAsymphony SP system (Qiagen, Germany) according to the manufacturer's instructions.

Paired tumor samples used in clinical diagnosis, formalin-fixed and paraffin-embedded (FFPE) tissue blocks or cytological specimens, was used for comparison and are hereafter referred to as tumor samples. Tumors were staged and histologically classified according to the guidelines of the International Association for the Study of Lung Cancer and World Health Organization 
nomenclature, respectively (Detterbeck et al. 2017; Travis et al. 2004). DNA was extracted using FFPE DNA Purification Kit on the MagLEAD 12gc (Precision System Science, Germany) or with QIAamp DNA FFPE Tissue kit on the Qiacube (Qiagen). RNA was extracted using FFPE Total RNA Purification Kit (Exscalebio, Sweden). Concentration of extracted DNA from plasma, tissue or cytology was measured using Qubit dsDNA HS assay kit (Thermo Fisher, USA, MA) and RNA from tissue or cytology with Qubit RNA HS assay kit (Thermo Fisher) with Qubit 2.0 Fluorometer (Thermo Fisher).

\section{Library preparation and sequencing}

Library preparation of cfDNA samples was performed with the AVENIO ctDNA Surveillance kit (Roche Diagnostics, Rotkreuz, Switzerland). Libraries were generated from 9.5 to $50 \mathrm{ng}$ of cfDNA according to the protocol provided by the manufacturer. Concentration and fragment length was measured with dsDNA HS assay kit (Thermo Fisher) on Qubit 2.0 Fluorometer (Thermo Fisher) and High sensitivity D5000 kit (Agilent, USA, CA) on 4200 TapeStation Instrument (Agilent), respectively. Generated libraries were sequenced on the Illumina NextSeq 550Dx using the High output kit v2 300-cycles (Illumina, San Diego, CA, USA) to a unique molecular depth of $>500 \times$.

DNA and RNA from tumor samples were analyzed with Ion AmpliSeq Colon and Lung Cancer Research Panel v2 (Thermo Fisher) consisting of 92 amplicons (size range 52-138 bp) from 22 genes and the Oncomine Solid Fusion transcript kit (Thermo Fisher) including four fusion transcripts, respectively (https://www.thermofish er.com/se/en/home/clinical/diagnostic-testing/condi tion-disease-diagnostics/oncology-diagnostics/oncom ine-solid-tumour-kits.html; Laurent-Puig et al. 2016). Library and template preparation was performed on the Ion Chef System (Thermo Fisher) and sequenced on the Ion Torrent systems, either Ion PGM or Ion GeneStudio S5 Prime. For sequencing, Ion 316/318 Chip Kit V2 BC or Ion 520/530 Chip Kit was used for PGM and S5 Prime, respectively.

When tumor samples were insufficient for variant analysis with NGS, data was retrieved from clinical data repositories consisting of qPCR and Fluorescent in situ hybridization (FISH). Where tumor samples were insufficient for analysis with both DNA and RNA NGS, DNA NGS was prioritized. The collective data from variant analysis on tumor samples is further referred to as clinical variant analysis data.

A subset of tumor samples $(n=24)$, where material was still available, was analyzed with the AVENIO FFPE Surveillance kit (Roche Diagnostics) according to protocol, with the concentration and fragment length measured as for the cfDNA libraries.

\section{Mapping and variant calling}

For cfDNA and tumor samples run with AVENIO assays, variants were called using the AVENIO Oncology Analysis software (version 2.0.0, Roche Diagnostics) using default settings, based on references (Newman et al. 2014, 2016; Talevich et al. 2016).

The Torrent Mapping Alignment Program (TMAP) within the Torrent Suite software version 5.10 (Thermo Fisher) was used to map sequences against the human reference genome (hg19). Default reporting of mutations was used with the addition of allowing for multiple nucleotide variant, multiple nucleotide polymorphism, and complex variants. Mutations were annotated with the Oncomine variant annotation (Thermo Fisher) within the Ion Reporter software v5.10 (Thermo Fisher). The lowest allowed depth of coverage was set to $1200 \times$ and variants were only considered if the allele frequency (AF) was $>5 \%$ in a tumor sample, with the exception of known hotspot genes with a cutoff of $3 \%$. If a hotspot variant appeared below cutoff, NGS was rerun with a new tissue or cytology section and included in the analyses if concordant.

For clinical interpretation of cfDNA and tumor samples run with AVENIO assays, NAVIFY ${ }^{\circledR}$ Mutation Profiler (version 1.1.1, Roche diagnostics) was used, which integrates data from Catalogue of Somatic Mutations in Cancer (COSMIC), The Cancer Genome Atlas (TCGA), ClinVar and Exome Aggregation Consortium (ExAC) for classification (Bamford et al. 2004; Yaung et al. 2020). For tumor samples run with Ion Ampliseq or Oncomine Solid fusion kit, the variant caller file was also run through NAVIFY $^{\circledR}$ Mutation Profiler for verification of consistency and additional interpretation of variants. Variants present in ExAC with a frequency $>0.01$ were filtered out. Clinically relevant variants were defined in databases and lung cancer studies (Hagemann et al. 2015; Frampton et al. 2015; Kobayashi et al. 2015; https://www.mycan cergenome.org/; Chakravarty et al. 2017) and included (variants targetable for treatment are marked with an asterisk): BRAF codon 600*; EGFR exon 19 deletions", exon 20 insertions, T790M*, codon 719*, exon 18 deletion E709-T710delinsD, A763_Y764insFQEA*, S768I* (exon 20), exon 19 insertions, C797S (exon 20), L858R*, codon 851 and 861* (exon 21); ERBB2 exon 20 insertions; KRAS codon 12, 13 and 61; NRAS codon 12, 13 and 61; PIK3CA codon 542, 545 and 1047 and in MET exon 14 splice variants* and deletions", Y1003*, D1010*; as well as fusions with $A L K^{*}, R E T^{*}$ and $R O S 1^{*}$. All KRAS variants in codon 12 and 13 were considered clinically relevant in 
accordance with Swedish guidelines, albeit variable prognostic value.

\section{Statistics}

Linear regression was used for association between sequencing performance and DNA input. Mutational load was defined as the total number of variants detected in plasma for each patient. Mann-Whitney U-test's and Kruskal-Wallis test's was used for analyzing differences regarding mutational load and AF between two and several groups, respectively.

Variant agreement between cfDNA and tumor samples was evaluated using Fisher's exact test, with tumor samples as reference. Variants detected by at least one of the variant analysis methods employed, with an $\mathrm{AF} \leq 0.01$ in ExAC and classified as pathogenic in COSMIC, were considered. Tumor samples with at least one concordant variant in cfDNA were considered concordant.

The impact of the number of variants $(0-3$ vs $4-9$ vs 10-22) on overall survival was estimated using Log-rank test and Cox proportional hazards, both by univariate and multivariate analysis. Covariates included stage (IIIIa vs IIIb-IV) and Eastern cooperative oncology group performance status (ECOG PS; 1, 2, 3-4). All variables fulfilled the assumption of proportional hazards. Survival analysis was visualized using Kaplan Meier survival estimate.

P-values $<0.05$ were considered statistically significant. Statistical analyses were performed using IBM SPSS statistics version 25.0 (SPSS Inc. Chicago, IL) and R version 4.0.2 with packages ggplot2 version 3.3.2 and GenVisR version 1.20.0 (Wickham 2016; Skidmore et al. 2016).

\section{Results}

\section{Cohort characteristics}

The cohort consisted of 77 study participants attending the lung clinic for investigation of lung cancer where a plasma sample was available for cfDNA variant analysis with NGS (Table 1, Additional file 1: Table S1). One cfDNA plasma sample was excluded due to $<500 \times$ unique molecular depth (UMD). Participants were diagnosed with NSCLC (AC; $\mathrm{n}=37$ ), NSCLC squamous cell carcinoma (SqCC; $n=15)$, SCLC $(n=8)$ or benign lung disease (controls; $\mathrm{n}=16$ ).

The median age for lung cancer patients $(n=60)$ was 72 years, with a slightly higher proportion of males $(53.3 \%, \mathrm{n}=32)$ and former smokers $(48.3 \%, \mathrm{n}=29)$ compared to never $(20.0 \%, \mathrm{n}=12)$ and current smokers $(31.7 \%, \mathrm{n}=19)$. Stage IIIb-IV disease was observed in $60.7 \%(n=41)$ of cases, and the most common first line treatment was chemotherapy $(38.3 \%, \mathrm{n}=23)$.

Control patients with benign lung disease $(n=16)$ had a median age of 75 years, with $56.3 \%(n=9)$ being males.
Smoking status was evenly distributed between never and former smokers $(37.5 \%, \mathrm{n}=6)$, with less current smokers $(25.0 \%, \mathrm{n}=4)$.

\section{Variant agreement between plasma and tumor samples}

This study used real-world clinical data and patients were included based on the availability of a cfDNA plasma sample, regardless if the quality or quantity of the tissue or cytology sample was sufficient for variant analysis. However, for agreement, a variant had to be detected in a tumor sample in order to be included. Tumor sample NGS data was available for DNA and RNA variant analysis in $86.7 \%(\mathrm{n}=52)$ and $52.5 \%(\mathrm{n}=31)$ of the cases, respectively. When tumor material was insufficient for NGS, DNA and RNA variant analysis was performed with qPCR $(4.9 \%, \mathrm{n}=3)$ and FISH $(27.9 \%, \mathrm{n}=17)$, respectively. Collectively, this is referred to as clinical variant analysis data. Tumor sample was insufficient for variant analysis in $8.3 \%(n=5)$ of the cases. Twenty-four samples had tumor sample available for further analysis with the AVENIO surveillance panel, where five tumor samples were excluded due to low exon coverage uniformity $(<40 \%$ of exons had $>300 \times$ coverage). In the control group, six patients had tissue available for DNA variant analysis (37.5\%). A flow chart of methods used for variant analysis in tumor samples in the study cohort is shown in Fig. 1.

\section{Agreement in all analyzed variants}

For tumor samples with clinical variant analysis data available, the number of variants ranged from 1 to 2 (Additional file 2: Full VCF data). Across histologies, variants were detected in $22 \mathrm{ACs}, 7 \mathrm{SqCCs}$ and 1 of the SCLC tumor samples. For the 19 samples analyzed with AVENIO surveillance panel there was an addition, to the samples already positive in clinical variant analysis, of 3 ACs and $2 \mathrm{SqCCs}$ with variants detected. Mean UMD of tumor samples analyzed with AVENIO surveillance panel was $1589 \times(n=19$, range $309-3261 \times)$ and the number of detected variants ranged from 1 to 12 (median 4). In total, 35 patients had variants detected in tumor samples and were consequently eligible for agreement analysis. The overall variant agreement of tumor and plasma samples was $62.9 \%(22 / 35)$, where $54.3 \%(19 / 35)$ had a complete agreement in all variants detected.

The AF of concordant and discordant variants in tumor samples were highly overlapping and ranged from 3.2 to $68 \%$ (median 18\%) for concordant variants, and from 5.5 to $68 \%$ (median 20\%) for discordant variants, respectively. Tumor sample variants that were concordant in cfDNA ranged from 0.07 to $16.93 \%$ (median $1.9 \%$ ) in AF. The concordant variant with the lowest $\mathrm{AF}$ in plasma was $0.07 \%$ in the $B R A F$ gene. 
Table 1 Cohort characteristics

\begin{tabular}{|c|c|c|c|c|}
\hline Characteristic & $\begin{array}{l}\text { Lung cancer } \\
n(\%)\left(n=60^{b}\right)\end{array}$ & $\begin{array}{l}\text { Controls } \\
n(\%)(n=16)^{d}\end{array}$ & $\begin{array}{l}\text { Lung cancer vs controls, } \\
\text { p-value }\end{array}$ & $\begin{array}{l}\text { Total N (\%) } \\
(\mathrm{N}=76)\end{array}$ \\
\hline Age & & & 0.126 & \\
\hline Median & 72 & 75 & & 72 \\
\hline Range & $39-85$ & $49-84$ & & $39-85$ \\
\hline Sex & & & 0.835 & \\
\hline Male & $32(53.3)$ & $9(56.3)$ & & $40(52.6)$ \\
\hline Female & $28(46.7)$ & $7(43.8)$ & & $36(47.4)$ \\
\hline Smoking & & & 0.391 & \\
\hline Never & $12(20.0)$ & $6(37.5)$ & & $19(25.0)$ \\
\hline Former & $29(48.3)$ & $6(37.5)$ & & $34(44.7)$ \\
\hline Current & $19(31.7)$ & $4(25.0)$ & & $23(30.3)$ \\
\hline \multicolumn{5}{|l|}{ Histology } \\
\hline$A C$ & $37(61.7)$ & & & $37(48.7)$ \\
\hline $\mathrm{SqCC}$ & $15(25.0)$ & & & 15 (19.7) \\
\hline $\mathrm{SCLC}$ & $8(13.3)$ & & & $8(10.5)$ \\
\hline Controls & & & & $16(21.1)$ \\
\hline \multicolumn{5}{|l|}{ Stage } \\
\hline |-||la & $19(31.7)$ & & & \\
\hline IIIb-IV & $41^{c}(68.3)$ & & & \\
\hline \multicolumn{5}{|l|}{ ECOG PS } \\
\hline PS 0 & $15(25.0)$ & & & \\
\hline PS 1 & $24(40.0)$ & & & \\
\hline PS 2 & $12(20.0)$ & & & \\
\hline PS $3-4^{a}$ & $9(15.0)$ & & & \\
\hline \multicolumn{5}{|l|}{ Treatment } \\
\hline Surgery & $9(15.0)$ & & & \\
\hline Radiation & $9(15.0)$ & & & \\
\hline Chemotherapy & $23(38.3)$ & & & \\
\hline Targeted therapy or immunotherapy & $10(16.7)$ & & & \\
\hline Best supportive care/no treatment & $9(15.0)$ & & & \\
\hline
\end{tabular}

Controls consisted of patients referred to the lung clinic with the suspicion of lung cancer but later diagnosed with different benign lung diseases

AC adenocarcinoma, SqCC squamous cell carcinoma, SCLC small cell lung cancer, ECOG PS Eastern cooperative oncology group performance status

${ }^{\text {a }}$ One patient had PS 4

${ }^{b}$ One cfDNA sample was excluded due to low unique molecular depth

${ }^{\mathrm{c}}$ Five patients had stage IIIb

${ }^{d}$ Controls with benign lung disease were diagnosed with different types of inflammation, fibrosis and noduli in and around the lung tissue

Variant agreement significantly differed with stage, where patients with stage IIIb-IV disease were 10.8 times more likely to display a concordant result compared to stage I-IIIa (stage I-IIIa, 25.0\%, 3/12; and stage IIIb-IV, 82.9\%, 19/23; p =0.002). There was no difference in agreement between $\mathrm{AC}$ and SqCC (15/25 and 7/9, $\mathrm{p}=0.23)$. Several mutations below the detection threshold of $5 \%$ for tumor samples were also confirmed in cfDNA (data not shown). In the control group, 6 patients had tissue available for mutational analysis where one of the patients had two mutations observed in tissue that were not concordant with cfDNA.

\section{Agreement in clinically relevant variants}

For assessment of variant agreement between plasma and tumor samples in clinically relevant genes; hotspot variants in EGFR (7), KRAS (11), and PIK3CA (1), and fusions in $A L K$ (3) and ROS1 (1) were detected in tumor samples. All had one clinically relevant variant and agreement was therefore evaluated in a binary mode (Table 2).

The overall agreement between tumor and plasma samples for clinically relevant variants was $65.2 \%(15 / 23)$. As with the agreement considering all variants, a concordant result in clinically relevant genes was significantly more common with stage IIIb-IV disease, where neither of the 


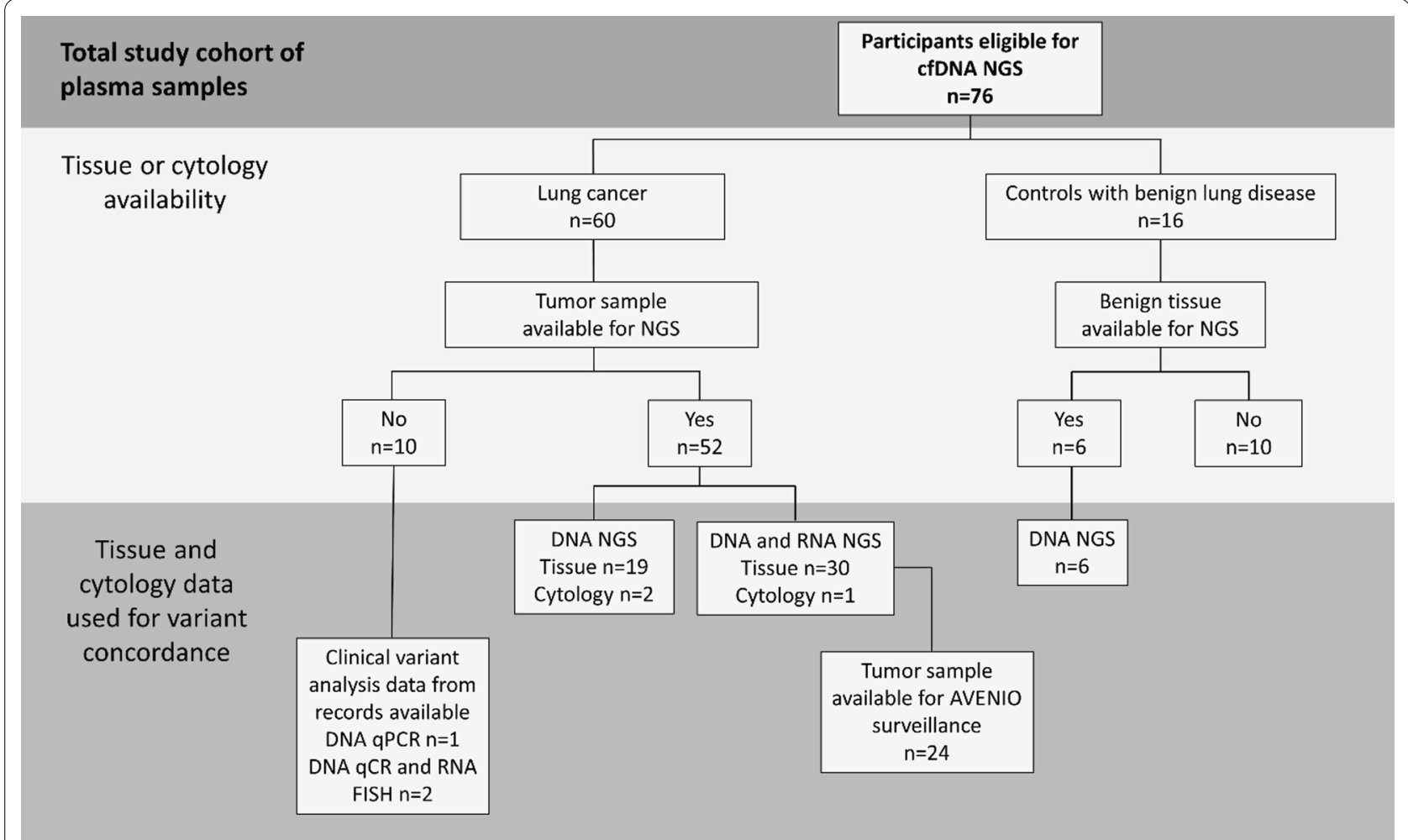

Fig. 1 Inclusion for variant agreement between tumor and plasma samples. The total study cohort consisted of patients with cfDNA AVENIO NGS performed $(n=77)$. One cfDNA plasma sample was excluded due to too low unique molecular depth $(<500 \times)$. For agreement between tumor or benign lung tissue and paired plasma, clinical variant analysis data from DNA and RNA NGS was retrieved. When tumor tissue or cytology was insufficient for NGS, clinical variant analysis data was retrieved from qPCR or FISH for DNA or RNA, respectively. A subset of patients $(n=24)$, where additional tumor material was available, was also analyzed with the corresponding AVENIO kit as for plasma

patients with stage I-IIIa $(0 / 6)$ had a concordant result, compared to $88.2 \%$, of patients with a stage IIIb-IV disease $(15 / 17, \mathrm{p}<0.001)$. One patient had a targetable $E G F R$ variant detected in plasma that was not detected in the tumor sample. Plasma detected and tumor sample absent variants were also observed in two other patients, harboring one $K R A S$ and one $B R A F$ mutation, respectively.

A total of 7 lung cancer patients had insufficient tumor material for mutational analysis, where one patient had a detectable KRAS mutation in cfDNA. Tumor material was also unavailable in 10 of the controls, with two having detectable variants in $K R A S$, of which one was a clinically relevant variant (p.Gly12Ala, AF: 0.06\%). Four tumor samples with confirmed fusions from clinical variant analysis, three in $A L K$ and one in ROS1, were further analyzed with AVENIO FFPE, where neither of the fusions were detected. In contrast, two of the four fusions were detected in cfDNA.

\section{Variant characteristics in plasma}

The entire cohort of plasma samples $(n=76)$ was analyzed using the AVENIO surveillance panel and variant characteristics in plasma was further explored distinct from tumor sample data. The mean UMD of plasma samples was $4871 \times(n=76$, range $713 \times-9243 \times)$. Input of cfDNA was associated with UMD ( $<<0.001)$, but not with overall number of detected variants $(\mathrm{p}=0.878)$, also referred to as mutational load.

The median number of variants detected in cfDNA was 5 for AC, 9 for SqCC, 6 for SCLC and 3 for controls with benign lung disease (Fig. 2A, Additional file 2: Full VCF data). There were significantly fewer variants detected in control patients compared to patients with lung cancer (median 3 vs $6, p=0.011$ ), but there was no significant difference between the different histologies of lung cancer $(p=0.465)$. There was a significantly higher mutational load in patients with stage IIIb-IV disease compared to patients with stage I-IIIa disease (median 7 vs 4 variants, $\mathrm{p}=0.017$; Fig. $2 \mathrm{~B}$ ) and to controls (median 7 vs 3 variants, $p=0.003$; Fig. $2 \mathrm{~B}$ ). There was a difference in mutational load regarding smoking status between never smokers and current/former smokers ( 4 variants vs 6 variants, $\mathrm{p}=0.028$ ). In a logistic regression, accounting for smoking status, an 
Table 2 Variant agreement between tumor and plasma samples in variants detected in clinically relevant genes

\begin{tabular}{|c|c|c|c|c|c|c|}
\hline \multirow[t]{2}{*}{ Study ID } & \multirow[t]{2}{*}{ Gene } & \multirow[t]{2}{*}{ Variant } & \multirow{2}{*}{$\begin{array}{l}\text { Tumor cell content } \\
\text { (\%) }\end{array}$} & \multirow{2}{*}{$\begin{array}{l}\text { Input cfDNA } \\
\text { (ng) }\end{array}$} & \multicolumn{2}{|l|}{$\mathrm{AF}(\%)$} \\
\hline & & & & & Tumor samples & cfDNA \\
\hline \multicolumn{7}{|c|}{ Concordant variants } \\
\hline 4 & KRAS & p.Gly12Cys & 50 & 37.3 & 52.85 & 3.06 \\
\hline 5 & KRAS & p.Gly12Val ${ }^{\text {b }}$ & 80 & 27.0 & NA & 2.09 \\
\hline 9 & EGFR & p.Glu746_Ala750del & 25 & 11.9 & 45.35 & 5.11 \\
\hline 18 & KRAS & p.Gly12Cys & 30 & 50.0 & 26.93 & 1.02 \\
\hline 25 & EGFR & p.Leu747_Pro753delinsSer & 20 & 50.0 & 45.83 & 2.28 \\
\hline 29 & PIK3CA & p.Glu545Lys & 20 & 44.7 & 20.41 & 8.10 \\
\hline 35 & ALK;EML4 & Fusion & 10 & 20.0 & NA & NA \\
\hline 42 & KRAS & p.Gly12Cys & 70 & 47.0 & 46.65 & 5.92 \\
\hline 51 & KRAS & p.Gly13Asp & 30 & 33.4 & 15.38 & 0.13 \\
\hline 52 & KRAS & p.Gly13Asp & 30 & 27.2 & 31.96 & 3.22 \\
\hline 55 & EGFR & p.Leu747_Glu749del & 20 & 20.5 & 39.53 & 5.80 \\
\hline 67 & KRAS & p.Gly12Cys & 10 & 50.0 & 19.01 & 3.38 \\
\hline 70 & ALK;EML4 & Fusion $^{c}$ & 10 & 13.9 & NA & NA \\
\hline 72 & EGFR & p.Leu858Arg & $N A^{a}$ & 50.0 & 1.65 & 0.19 \\
\hline 75 & KRAS & p.Gly12Cys & $<10$ & 43.9 & 11.05 & 0.52 \\
\hline \multicolumn{7}{|c|}{ Discordant variants } \\
\hline 1 & EGFR & p.Glu746_Ala750del ${ }^{b}$ & 60 & 19.8 & NA & - \\
\hline 7 & KRAS & p.Gly12Ala & 70 & 15.6 & 12.43 & - \\
\hline 21 & EGFR & p.Glu746_Ser752delinsVal & 20 & 22.7 & 27.71 & - \\
\hline 32 & ROS1 & Fusion $^{c}$ & 80 & 25.8 & NA & - \\
\hline 33 & EGFR & p.Glu746_Ala750del & 30 & 29.1 & 62.85 & - \\
\hline 43 & KRAS & p.Gly12Cys & 5 & 35.7 & 8.15 & - \\
\hline 60 & ALK;EML4 & Fusion & 25 & 22.5 & NA & - \\
\hline 73 & KRAS & p.Gly12Ala & 20 & 38.1 & 15.08 & - \\
\hline
\end{tabular}

All variants detected in clinically relevant genes in tumor samples are included and each line represents one case

increase in mutational load resulted in a $24 \%$ higher odds of having a stage IIIb-IV tumor $(\mathrm{p}=0.012,95 \% \mathrm{CI}$ 1.05-1.47).

AF of variants detected in plasma cfDNA ranged from 0.03 to $19.44 \%$ (median $0.25 \%$ ). There was no association between $\mathrm{AF}$ and smoking status, $(0.26 \%$, never vs $0.32 \%$, former/current, $\mathrm{p}=0.237)$. There was no difference in AF between lung cancer and controls $(p=0.537)$ or between different histologies $(p=0.838)$ and stage $(p=0.287)$. Some cfDNA samples had several single nucleotide polymorphisms present at low AF. This was more common in SCLC where three samples had $50 \%$ of the low AF variants present in EXAC at high frequencies.

The most commonly mutated genes in the total lung cancer patient cohort were TP53 and EGFR, with variants in $36.7 \%(22 / 60)$ of participants each (Fig. 3A). When analyzing different histologies separately,
TP53 remained the most mutated gene in both SqCC $(46.7 \%, 7 / 15)$ and SCLC $(50.0 \%, 4 / 8)$, but the second most common in AC $(29.7 \%, 11 / 37)$. The second most common genes with variants in SqCC were KIT and EGFR in $33.3 \%(5 / 15)$ of cases each, where neither of the detected $E G F R$ variants were associated with response to EGFR tyrosine kinase inhibitors. For AC, $E G F R$ was the most commonly mutated gene present in $37.8 \%(14 / 37)$ of cases, out of which $35.7 \%(5 / 14)$ of cases were targetable. The third most common gene with variants was $K R A S(24.3 \%, 9 / 37)$ and the fourth was shared by ERBB2 and $M E T(18.9 \%, 7 / 37)$. The SCLC group was too small to examine further. In the control group, BRCA2 and NPAP1 were the most commonly mutated genes present in $18.8 \%(3 / 16)$ of cases each (Fig. 3B). Variants in both BRCA1 and BRCA2 were present across all histologies of lung cancer 

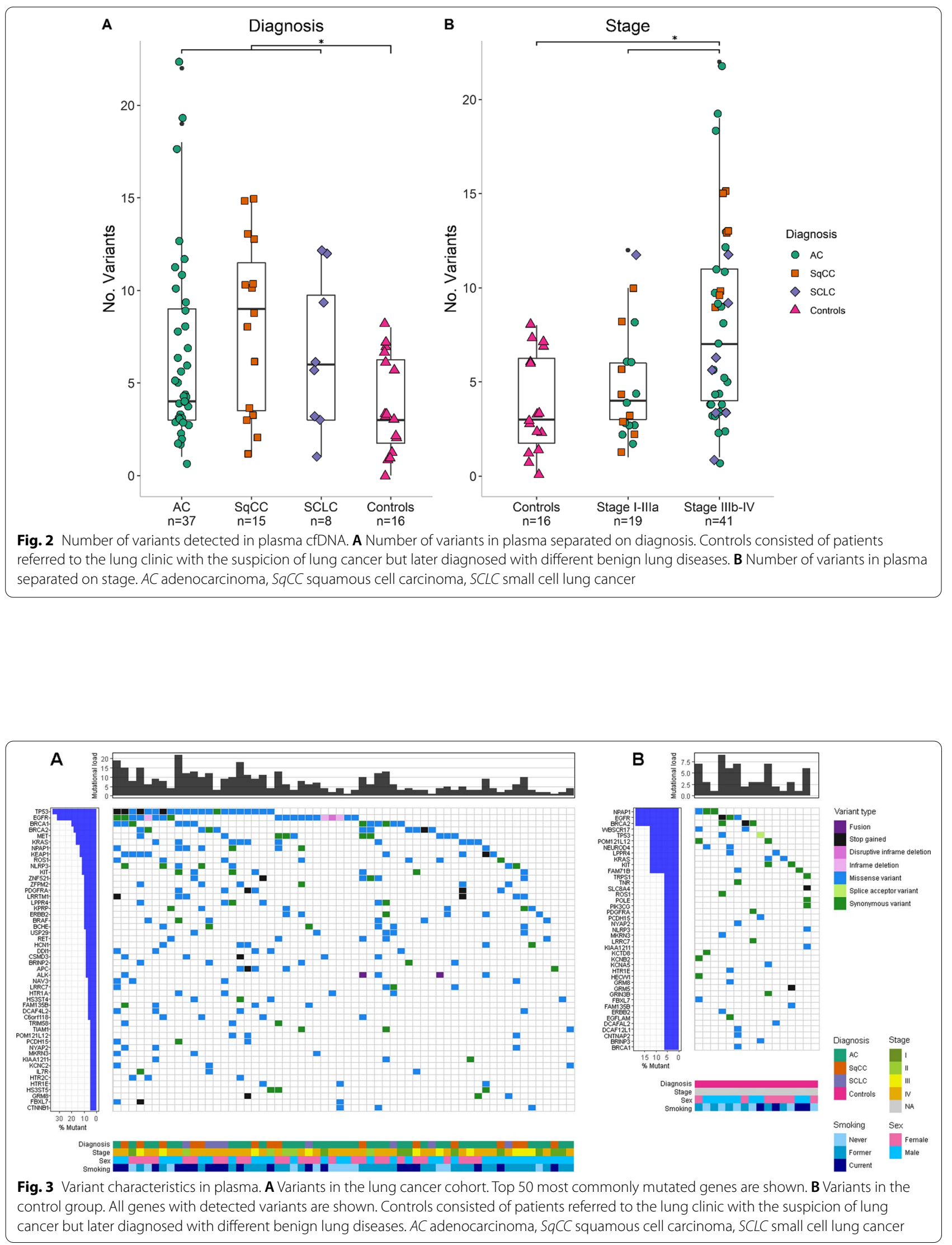
and controls, with a prevalence of $20.0 \%(12 / 37)$ and $6.3 \%$ (1/16) for BRCA1, and $18.3 \%$ (11/37) and $18.8 \%$ (3/16) for BRCA2 in lung cancer patients and controls, respectively. The remaining variants were distributed at low frequencies across numerous genes. The AVENIO software detects copy number variants (CNVs) in EGFR, MET and ERBB2 genes. In total, four positive CNVs were detected. Two patients, one with AC and one with SCLC, had CNVs in EGFR while a third patient with SCLC had CNVs in both EGFR and MET; corresponding to $25 \%(2 / 8)$ and $2.6 \%(1 / 37) \mathrm{CNV}$ positivity in SCLC and AC subgroups, respectively.

For survival analysis, patients with lung cancer were divided into three groups based on mutational load in cfDNA: $0-3$ variants $(\mathrm{n}=20) ; 4-9$ variants $(\mathrm{n}=22)$; and $\geq 10$ variants $(n=18)$. In a univariate model, cfDNA mutational load was significantly associated with overall survival ( $p=0.0098$, log rank test; Fig. 4). This association persisted between patients with $0-3$ and 10-22 variants in a multivariate model adjusted for stage and ECOG PS (HR: 3.64, 95\% CI 1.37-9.67, $\mathrm{p}=0.009$; Table 3 ).

\section{Discussion}

In the present study, we investigated the use of cfDNA in plasma as a primary source for molecular variant diagnostics in a cohort of patients with lung cancer. We found that the mutational load in cfDNA was associated with overall survival in a multivariate model. In addition, a high agreement of variants between tumor and plasma samples in patients presenting with stage IIIb-IV disease was observed, but also a number of variants in plasma that could not be confirmed in tumor samples.

The most frequently mutated gene in plasma was TP53 with a frequency of $33.8 \%$ in the total cohort and an enrichment in SqCC and SCLC (46.7\% and 50.0\%, respectively), compared to $\mathrm{AC}$ (31.6\%). This is consistent with previous findings where variants in TP53 is more common in SqCC compared to AC (Gibbons et al. 2014). Also consistent with the literature is the presence of EGFR variants in $36.8 \%$ in $\mathrm{AC}$, where $13.5 \%$ in total was targetable by tyrosine kinase inhibitors. However, 33.3\% EGFR positivity in $\mathrm{SqCC}$ in our cohort is aberrantly high since this histology is, almost exclusively, EGFR negative (Rekhtman et al. 2012). However, neither of these

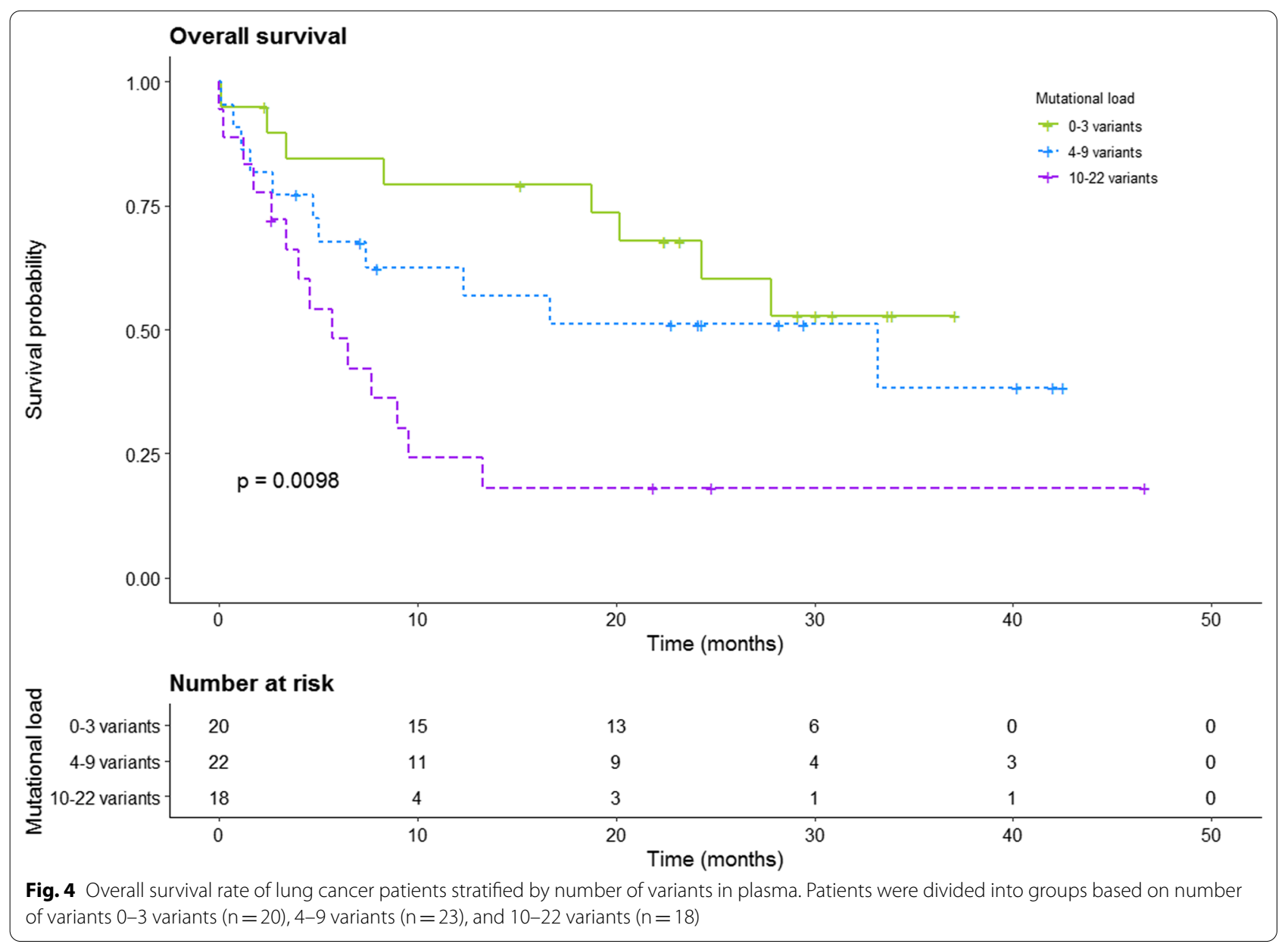


Table 3 Cox regression models of mutational load on survival

\begin{tabular}{|c|c|c|c|c|c|c|}
\hline \multirow[t]{2}{*}{ Variable } & \multicolumn{3}{|c|}{ Univariate model } & \multicolumn{3}{|c|}{ Multivariate model } \\
\hline & $\mathrm{HR}$ & $95 \% \mathrm{Cl}$ & p-value & HR & $95 \% \mathrm{Cl}$ & p-value \\
\hline \multicolumn{7}{|l|}{ Mutational load } \\
\hline $0-3$ variants & 1 (ref) & & & 1 (ref) & & \\
\hline $4-9$ variants & 1.58 & $0.63-3.93$ & 0.328 & 1.95 & $0.72-5.32$ & 0.192 \\
\hline $10-22$ variants & 3.51 & $1.45-8.52$ & 0.006 & 3.64 & $1.37-9.67$ & 0.009 \\
\hline \multicolumn{7}{|l|}{ Stage } \\
\hline I-IIIa & & & & 1 (ref) & & \\
\hline IIIb-IV & & & & 1.898 & $0.70-5.11$ & 0.205 \\
\hline \multicolumn{7}{|l|}{ ECOG PS } \\
\hline PS 0 & & & & 1 (ref) & & \\
\hline PS 1 & & & & 1.19 & $0.39-3.70$ & 0.76 \\
\hline PS 2 & & & & 3.30 & $1.06-10.31$ & 0.04 \\
\hline PS 3-4 & & & & 76.05 & $14.95-386.85$ & 0.000 \\
\hline
\end{tabular}

ECOG PS Eastern cooperative oncology group performance status, $\mathrm{HR}$ hazard ratio, $\mathrm{Cl}$ confidence interval

variants were targetable and could be explained as possible passenger mutations.

In this study, mutational load, defined as the total number of variants detected in cfDNA, was used as a simplified version of TMB. We found that mutational load in cfDNA plasma samples was associated to prognosis. Mutational load in tissue, measured by TMB, has been associated to prognosis of lung cancer previously, but international consensus guidelines concerning choice of sequencing method and cutoff values have not been reached. TMB has not been as thoroughly investigated in cfDNA and, as previously mentioned, several studies have reported a varying agreement in TMB between tissue and cfDNA (Koeppel et al. 2017; Gandara et al. 2018; Andrew et al. 2017). Müller et al. found a high correlation of TMB results when using whole genome sequencing and targeted sequencing using the CAPP-seq panel of 134 genes, a smaller predecessor of the panel used herein (Muller et al. 2017). In our study, the input amount of cfDNA was not associated with the total number of variants detected, contrary to a previous report (Koeppel et al. 2017). Despite the scattered results of cfDNA TMB, it may still be clinically useful since TMB has shown promising results for predicting response and prognosis of lung cancer patients treated with immunotherapy (Gandara et al. 2018; Khagi et al. 2017). Regarding other cancer types, one study on breast cancer patients treated with surgery and radiotherapy has shown an association between the number of cfDNA variants and relapse-free survival (Kujala et al. 2020). Jaiswal et al. (2014) found that the presence of a somatic mutation in peripheral blood cells was associated with an increase in all-cause mortality. To our knowledge, the present study is the first to report an association between the number of variants in plasma and prognosis for patients with lung cancer, irrespective of treatment.

We found a higher variant agreement between plasma and tumor samples when analyzing stage IIIb-IV disease, compared to stage I-IIIa disease, which is consistent with previous reports (Jiang and Yao 2020; Karlovich and Sun 2016). An increased likelihood of detecting a variant in an advanced stage disease is related to its elevated ability to shed DNA into the bloodstream. Apart from stage, the shedding ability has been associated to tumor size, metastatic location and genomic subtype (Lam et al. 2020; Cho et al. 2020). This adds further information for concordant and discordant variants while, unfortunately, this cohort did not have enough sample size to evaluate this further.

At present, targeted drugs are only prescribed to patients with a IIIb-IV stage disease, and is consequently in a greater need of variant analysis compared to patients with stage I-IIIa disease (https://kunsk apsbanken.cancercentrum.se/diagnoser/lungcancer/ vardprogram/). Our result of $82.9 \%$ total agreement, and $88.2 \%$ agreement of clinically relevant variants, between plasma and tumor samples in stage IIIb-IV disease is given further clinical value when considering the combined fail rate of obtaining a tissue biopsy and it in turn having sufficient quality for NGS analysis. As previously mentioned, an advanced disease is highly associated with an overall decline in general health status, which also corresponds to an increased fail rate for tissue sampling. In previous studies, this fail rate has been $19-51 \%$ and considering the fraction of patients with NSCLC having a targetable variant, it is 
highly likely that several patients eligible for targeted therapy are wrongly omitted (Douillard et al. 2014; Aggarwal et al. 2019; Meric-Bernstam et al. 2015; Hellmann et al. 2018; Thompson and Troxel 2016). In this study, the fail rate of tissue sampling was much lower, but there was still one clinically relevant mutation in $K R A S$ detected in cfDNA corresponding to one of the insufficient tumor samples. In addition, three patients with tumor sample analysis results had clinically relevant variants detected in cfDNA that were undetectable in tumor samples. One of these patients had a targetable EGFR variant that would possibly have benefitted from targeted therapy. Even when disregarding the clinically relevant variants only detected in plasma, our results imply that for patients with stage IIIb-IV disease, to which the currently available targeted drugs are prescribed, nine out of ten patients would have sufficed with molecular variant diagnostics on plasma instead of tissue or cytology. Since plasma is a non-invasive and easily obtained tumor material source compared to a biopsy, a simple blood draw could have spared these patients from a painful attempt to repeat a biopsy with insufficient material for variant analysis.

In our cohort, several detected variants in plasma could not be verified in tumor samples when analyzing all variants. The number of detected variants in plasma was higher in patients with lung cancer compared to the control group in this study, suggesting a tumor origin for a large part of the variants. Non-verified variants in tumor samples could be due to tumor heterogeneity or they could originate from clonal hematopoiesis $(\mathrm{CH})$ or other non-tumor cells. Challenges with contribution of $\mathrm{CH}$ regarding variants in plasma has been reported previously where Liu et al. observed that $75 \%$ of variants detected in healthy controls older than 50 years originated from $\mathrm{CH}$. On the other hand, Coombs et al. (2017) reported that $25 \%$ of the plasma variants in patients with solid tumors originated from $\mathrm{CH}$ (Liu et al. 2019). Out of the nine most commonly mutated genes in $\mathrm{CH}$, accounting for the majority of $\mathrm{CH}$ contribution, the panel used in the present study only contained one, TP53. Contrary to previous reports, TP53 variants were not frequently detected in the control group in the present study $(\mathrm{Hu}$ et al. 2018; Fernandez-Cuesta et al. 2016). However, $B R C A$ genes appeared with a number of variants at low AF across several histologies, including controls, and were not classified as germline. This diverges from what has previously been reported, where to our knowledge only one case with a somatic $B R C A$ variant has been described in lung cancer, in a population with a high $B R C A$ variant prevalence, and any $B R C A$ variant presence in $\mathrm{CH}$ has not been reported (Kadouri et al. 2019). A lot is still unknown about the origin of variants in plasma and this is an intriguing area for future research to better understand the biological background in plasma.

Three cases with CNVs in EGFR and MET were detected in this study. One patient presented with an amplification of both EGFR and MET, which suggests an aneuploidy event on chromosome 7 (Zojer et al. 2000). This patient, and one with an amplification in EGFR only, were both diagnosed with SCLC. There have been no previous reports of amplifications in these genes in SCLC. In contrast, a decreased protein expression of EGFR in SCLC compared to AC has been reported (Cerny et al. 1986; Sobol et al. 1987; Niederst et al. 2015; Shi et al. 2016). The heterogeneity of lung cancer tumors is well known and the small biopsies obtained may not always reflect the entire tumor entity. Therefore, a tumor is not seldom classified with the dominant histology where combinations of NSCLC and SCLC have been reported (Babakoohi et al. 2013; Lei et al. 2020; Vogelstein et al. 2013). Thus, it is possible that the plasma sample may illustrate a part of the tumor that was not detected in the tumor sample.

This study attempted to mimic the clinical real-world situation, increasing the clinical relevance of our findings. When analyzing cfDNA it is important to be able to evaluate the non-tumor variant background noise, which we addressed by the inclusion of a control group of patients that were investigated for lung cancer. To perform a more specific filtering of non-tumor variants matched white blood cells or machine learning algorithms would have been more preferable (Chabon et al. 2020). However, the cost of performing a paired deep sequencing on larger genomic regions is a considerable disadvantage for health economic reasons and even though machine learning has shown promise, it is still in an early phase.

The limitations of this study is in great part its size and the disadvantages of having clinical real-world data, which includes a sparse amount and quality of the obtained tissue or cytology. Therefore, further analyses using the same panel as with cfDNA was only possible for a small subset of patients and it is possible that several more variants in cfDNA could have been confirmed if more tumor sample had been available. It is evident that cfDNA has a clear clinical value even though some optimization is still needed in order to make confident calls about tumor origin of specific variants on a broad spectrum NGS in plasma.

\section{Conclusion}

This study concludes that in the vast majority of advanced NSCLC patients a reliable variant analysis can be performed using liquid biopsy. The blood analysis provides additional molecular information to the tissue based analysis. This study also presents novel findings 
that the number of variants in plasma are associated with prognosis, possibly indicating a strategy for closer follow up on this crucial patient group. The data presented here could thereby aid effective personalized therapy for patients with lung cancer.

\section{Abbreviations}

AF: Allele frequency; cfDNA: Circulating cell-free DNA; ctDNA: Circulating tumor DNA; CH: Clonal hematopoiesis; CNVs: Copy number variants; NGS: Next generation sequencing; TMB: Tumor mutational burden; UMD: Unique molecular depth.

\section{Supplementary Information}

The online version contains supplementary material available at https://doi. org/10.1186/s10020-021-00331-1.

Additional file 1: Table S1. Cohort characteristics and cfDNA MPS quality. Controls consisted of patients referred to the lung clinic with the suspicion of lung cancer but later diagnosed with benign lung diseases. UMD: Unique molecular depth; PS: ECOG Performance status; AC: Adenocarcinoma; SqCC: Squamous cell carcinoma; SCLC: Small cell lung cancer.

Additional file 2. Full VCF data.

\section{Acknowledgements}

We wish to extend our appreciation to the Department of Laboratory Medicine, Molecular Diagnostics R\&D at Örebro University Hospital for facilities and laboratory equipment. We also wish to acknowledge our contacts at Roche Diagnostics for support with the workflow and bioinformatical analysis.

\section{Authors' contributions}

$A Q$ performed all laboratory work, interpreted results and wrote the draft. $A Q$, $\mathrm{BS}, \mathrm{JC}, \mathrm{Jl}$ and $\mathrm{GH}$ contributed to the formal analysis. $\mathrm{AQ}$ and $\mathrm{GH}$ contributed to study design and funding acquisition. BS, JC, JI, CK and GH revised the manuscript. All authors read and approved the final manuscript.

\section{Funding}

This work was supported by the Örebro County Council Research committee; the Lions fund for cancer research Uppsala-Örebro; Nyckelfonden-Örebro University Hospital Research Foundation; and the Uppsala-Örebro Regional research council.

\section{Availability of data and materials}

The cfDNA data can be found at the European Nucleotide Archive with Accession PRJEB45628. Variant Call Format files for all samples analyzed with NGS can be found in Additional file 2 attached to this article.

\section{Declarations}

\section{Ethics approval and consent to participate}

The study was approved by the regional ethics committee board in Uppsala (Dnr 2015-400) and patients gave written informed consent before inclusion.

\section{Consent for publication}

Not applicable.

\section{Competing interests}

The authors declare that the AVENIO reagents were partly funded by Roche Diagnostics, which also provided access to NAVIFY Mutation Profiler and gave bioinformatical support. Roche Diagnostics took no part in study design or interpretation of the results.

\section{Author details}

${ }^{1}$ Dept. of Laboratory Medicine, Örebro University Hospital, Södra Grev. Roseng., 70185 Örebro, Sweden. ${ }^{2}$ Faculty of Medicine and Health, Örebro
University, Örebro, Sweden. ${ }^{3}$ Dept. of Urology, Faculty of Medicine and Health, Örebro University, Örebro, Sweden. ${ }^{4}$ Dept. of Respiratory Medicine, Gävle Hospital, Gävle, Sweden. ${ }^{5}$ Centre for Research and Development Region Gävleborg/Uppsala University, Gävle, Sweden. ${ }^{6}$ Dept. of Immunology, Genetics and Pathology, Uppsala University, Uppsala, Sweden. ${ }^{7}$ School of Health Sciences, Örebro University, Örebro, Sweden.

Received: 25 January 2021 Accepted: 16 June 2021

Published online: 03 July 2021

\section{References}

Aggarwal C, Thompson JC, Black TA, Katz SI, Fan R, Yee SS, et al. Clinical implications of plasma-based genotyping with the delivery of personalized therapy in metastatic non-small cell lung cancer. JAMA Oncol. 2019;5(2):173-80. https://doi.org/10.1001/jamaoncol.2018.4305.

Babakoohi S, Fu P, Yang M, Linden PA, Dowlati A. Combined SCLC clinical and pathologic characteristics. Clin Lung Cancer. 2013;14(2):113-9. https:// doi.org/10.1016/j.cllc.2012.07.002.

Bamford S, Dawson E, Forbes S, Clements J, Pettett R, Dogan A, et al. The COSMIC (Catalogue of Somatic Mutations in Cancer) database and website. Br J Cancer. 2004;91(2):355-8. https://doi.org/10.1038/sj.bjc.6601894.

Brahmer J, Reckamp KL, Baas P, Crino L, Eberhardt WE, Poddubskaya E, et al. Nivolumab versus docetaxel in advanced squamous-cell non-small-cell lung cancer. N Engl J Med. 2015;373(2):123-35. https://doi.org/10.1056/ NEJMoa1504627.

Cancer. RCf. Swedish national care program for lung cancer. https://kunsk apsbanken.cancercentrum.se/diagnoser/lungcancer/vardprogram/.

Cerny T, Barnes DM, Hasleton P, Barber PV, Healy K, Gullick W, et al. Expression of epidermal growth factor receptor (EGF-R) in human lung tumours. $\mathrm{Br} J$ Cancer. 1986;54(2):265-9. https://doi.org/10.1038/bjc.1986.172.

Chabon JJ, Hamilton EG, Kurtz DM, Esfahani MS, Moding EJ, Stehr H, et al. Integrating genomic features for non-invasive early lung cancer detection. Nature. 2020;580(7802):245-51. https://doi.org/10.1038/ s41586-020-2140-0.

Chakravarty D, Gao J, Phillips SM, Kundra R, Zhang H, Wang J, et al. OncoKB: a precision oncology knowledge base. JCO Precis Oncol. 2017. https://doi. org/10.1200/PO.17.00011.

Cho MS, Park CH, Lee S, Park HS. Clinicopathological parameters for circulating tumor DNA shedding in surgically resected non-small cell lung cancer with EGFR or KRAS mutation. PLOS ONE. 2020;15(3):e0230622. https://doi. org/10.1371/journal.pone.0230622.

Coombs CC, Zehir A, Devlin SM, Kishtagari A, Syed A, Jonsson P, et al. Therapyrelated clonal hematopoiesis in patients with non-hematologic cancers is common and associated with adverse clinical outcomes. Cell Stem Cell. 2017;21(3):374-82.e4. https://doi.org/10.1016/j.stem.2017.07.010.

Davis AA, Chae YK, Agte S, Pan A, Simon NI, Taxter TJ, Behdad A, Carneiro BA, Cristofanilli M, Giles FJ. Comparison of tumor mutational burden (TMB) across tumor tissue and circulating tumor DNA (ctDNA). J Clin Oncol. 2017;35:e23028. https://doi.org/10.1200/JCO.2017.35.15_suppl.e23028.

Detterbeck FC, Boffa DJ, Kim AW, Tanoue LT. The eighth edition lung cancer stage classification. Chest. 2017;151(1):193-203. https://doi.org/10.1016/j. chest.2016.10.010.

Douillard JY, Ostoros G, Cobo M, Ciuleanu T, Cole R, McWalter G, et al. Gefitinib treatment in EGFR mutated caucasian NSCLC: circulating-free tumor DNA as a surrogate for determination of EGFR status. J Thorac Oncol. 2014;9(9):1345-53. https://doi.org/10.1097/JTO.0000000000000263.

Drilon A, Laetsch TW, Kummar S, DuBois SG, Lassen UN, Demetri GD, et al. Efficacy of larotrectinib in TRK fusion-positive cancers in adults and children. N Engl J Med. 2018;378(8):731-9. https://doi.org/10.1056/NEJMoa1714 448.

Fernandez-Cuesta L, Perdomo S, Avogbe PH, Leblay N, Delhomme TM, Gaborieau $\mathrm{V}$, et al. Identification of circulating tumor DNA for the early detection of small-cell lung cancer. EBioMedicine. 2016;10:117-23. https://doi. org/10.1016/j.ebiom.2016.06.032.

Food and Drug Administration. FDA/CEDR resources page. https://www.fda. gov/drugs/drug-approvals-and-databases/fda-approves-pembrolizu mab-adults-and-children-tmb-h-solid-tumors.

Frampton GM, Ali SM, Rosenzweig M, Chmielecki J, Lu X, Bauer TM, et al. Activation of MET via diverse exon 14 splicing alterations occurs in multiple 
tumor types and confers clinical sensitivity to MET inhibitors. Cancer Discov. 2015;5(8):850-9. https://doi.org/10.1158/2159-8290.CD-15-0285.

Gandara DR, Paul SM, Kowanetz M, Schleifman E, Zou W, Li Y, et al. Bloodbased tumor mutational burden as a predictor of clinical benefit in non-small-cell lung cancer patients treated with atezolizumab. Nat Med. 2018;24(9):1441-8. https://doi.org/10.1038/s41591-018-0134-3.

Gao Y, Zhang K, Xi H, Cai A, Wu X, Cui J, et al. Diagnostic and prognostic value of circulating tumor DNA in gastric cancer: a meta-analysis. Oncotarget. 2017;8(4):6330-40. https://doi.org/10.18632/oncotarget.14064.

Gibbons DL, Byers LA, Kurie JM. Smoking, p53 mutation, and lung cancer. Mol Cancer Res. 2014;12(1):3-13. https://doi.org/10.1158/1541-7786. MCR-13-0539.

Hagemann IS, Devarakonda S, Lockwood CM, Spencer DH, Guebert K, Bredemeyer AJ, et al. Clinical next-generation sequencing in patients with non-small cell lung cancer. Cancer. 2015;121(4):631-9. https://doi.org/10. 1002/cncr.29089.

Hellmann MD, Ciuleanu TE, Pluzanski A, Lee JS, Otterson GA, Audigier-Valette $C$, et al. Nivolumab plus ipilimumab in lung cancer with a high tumor mutational burden. N Engl J Med. 2018;378(22):2093-104. https://doi. org/10.1056/NEJMoa1801946.

Hu Y, Ulrich BC, Supplee J, Kuang Y, Lizotte PH, Feeney NB, et al. False-positive plasma genotyping due to clonal hematopoiesis. Clin Cancer Res. 2018;24(18):4437-43. https://doi.org/10.1158/1078-0432.CCR-18-0143.

Jaiswal S, Fontanillas P, Flannick J, Manning A, Grauman PV, Mar BG, et al. Agerelated clonal hematopoiesis associated with adverse outcomes. N Engl J Med. 2014;371(26):2488-98. https://doi.org/10.1056/NEJMoa1408617.

Jiang T, Zhai C, Su C, Ren S, Zhou C. The diagnostic value of circulating cell free DNA quantification in non-small cell lung cancer: a systematic review with meta-analysis. Lung Cancer. 2016;100:63-70. https://doi.org/10. 1016/j.lungcan.2016.06.013.

Jiang JAH, Yao L, et al. Concordance of genomic alterations by next-generation sequencing in tumor tissue versus cell-free dna in stage i-iv non-small cell lung cancer. J Mol Diagn. 2020;22(2):228-35. https://doi.org/10. 1016/j.jmoldx.2019.10.013.

Kadouri L, Rottenberg Y, Zick A, Hamburger T, Lipson D, Peretz T, et al. Homologous recombination in lung cancer, germline and somatic mutations, clinical and phenotype characterization. Lung Cancer. 2019;137:48-51. https://doi.org/10.1016/j.lungcan.2019.09.008.

Karlovich CGJ, Sun JM, et al. Assessment of EGFR mutation status in matched plasma and tumor tissue of NSCLC patients from a phase I study of rociletinib (CO-1686). Clin Cancer Res. 2016;22(10):2386-95. https://doi.org/ 10.1158/1078-0432.CCR-15-1260.

Khagi Y, Goodman AM, Daniels GA, Patel SP, Sacco AG, Randall JM, et al. Hypermutated circulating tumor DNA: correlation with response to checkpoint inhibitor-based immunotherapy. Clin Cancer Res. 2017;23(19):5729-36. https://doi.org/10.1158/1078-0432.CCR-17-1439.

Kobayashi Y, Togashi Y, Yatabe Y, Mizuuchi H, Jangchul P, Kondo C, et al. EGFR Exon 18 mutations in lung cancer: molecular predictors of augmented sensitivity to afatinib or neratinib as compared with first- or third-generation TKls. Clin Cancer Res. 2015;21(23):5305-13. https://doi.org/10.1158/ 1078-0432.CCR-15-1046.

Koeppel F, Blanchard S, Jovelet C, Genin B, Marcaillou C, Martin E, et al. Whole exome sequencing for determination of tumor mutation load in liquid biopsy from advanced cancer patients. PLoS ONE. 2017;12(11):e0188174. https://doi.org/10.1371/journal.pone.0188174.

Kujala J, Hartikainen JM, Tengstrom M, Sironen R, Kosma VM, Mannermaa A. High mutation burden of circulating cell-free DNA in early-stage breast cancer patients is associated with a poor relapse-free survival. Cancer Med. 2020. https://doi.org/10.1002/cam4.3258.

Lam VK, Zhang J, Wu CC, Tran HT, Li L, Diao L, et al. Genotype-specific differences in circulating tumor DNA levels in advanced NSCLC. J Thorac Oncol. 2020. https://doi.org/10.1016/j.jtho.2020.12.011.

Laurent-Puig P, Vaughn C, Lacroix L, M L. Ion AmpliSeq colon and lung cancer research Panel v2 and Ion AmpliSeq RNA fusion lung cancer research panel. Thermo Fisher Scientific; 2016.

Le DT, Uram JN, Wang H, Bartlett BR, Kemberling H, Eyring AD, et al. PD-1 blockade in tumors with mismatch-repair deficiency. $N$ Engl I Med. 2015;372(26):2509-20. https://doi.org/10.1056/NEJMoa1500596.

Lei Y, Feng H, Qiang H, Shang Z, Chang Q, Qian J, et al. Clinical characteristics and prognostic factors of surgically resected combined small cell lung cancer: a retrospective study. Lung Cancer. 2020;146:244-51. https://doi.org/10.1016/j.lungcan.2020.06.021.

Lin Z, Neiswender J, Fang B, Ma X, Zhang J, Hu X. Value of circulating cell-free DNA analysis as a diagnostic tool for breast cancer: a metaanalysis. Oncotarget. 2017:8(16):26625-36. https://doi.org/10.18632/ oncotarget.15775.

Liu J, Chen X, Wang J, Zhou S, Wang CL, Ye MZ, et al. Biological background of the genomic variations of cf-DNA in healthy individuals. Ann Oncol. 2019;30(3):464-70. https://doi.org/10.1093/annonc/mdy513.

Marchetti A, Palma JF, Felicioni L, De Pas TM, Chiari R, Del Grammastro $M$, et al. Early prediction of response to tyrosine kinase inhibitors by quantification of EGFR mutations in plasma of NSCLC patients. J Thorac Oncol. 2015;10(10):1437-43. https://doi.org/10.1097/JTO.0000000000 000643.

Meric-Bernstam F, Brusco L, Shaw K, Horombe C, Kopetz S, Davies MA, et al. Feasibility of large-scale genomic testing to facilitate enrollment onto genomically matched clinical trials. J Clin Oncol. 2015;33(25):2753-62. https://doi.org/10.1200/JCO.2014.60.4165.

Muller JN, Falk M, Talwar J, Neemann N, Mariotti E, Bertrand M, et al. Concordance between comprehensive cancer genome profiling in plasma and tumor specimens. J Thorac Oncol. 2017;12(10):1503-11. https:// doi.org/10.1016/j.jtho.2017.07.014.

My Cancer Genome. https://www.mycancergenome.org/.

Newman AM, Bratman SV, To J, Wynne JF, Eclov NC, Modlin LA, et al. An ultrasensitive method for quantitating circulating tumor DNA with broad patient coverage. Nat Med. 2014;20(5):548-54. https://doi.org/ 10.1038/nm.3519.

Newman AM, Lovejoy AF, Klass DM, Kurtz DM, Chabon JJ, Scherer F, et al. Integrated digital error suppression for improved detection of circulating tumor DNA. Nat Biotechnol. 2016;34(5):547-55. https://doi.org/10. 1038/nbt.3520.

Nie K, Jia Y, Zhang X. Cell-free circulating tumor DNA in plasma/serum of non-small cell lung cancer. Tumour Biol. 2015;36(1):7-19. https://doi. org/10.1007/s13277-014-2758-3.

Niederst MJ, Sequist LV, Poirier JT, Mermel CH, Lockerman EL, Garcia AR, et al. RB loss in resistant EGFR mutant lung adenocarcinomas that transform to small-cell lung cancer. Nat Commun. 2015;6:6377. https:// doi.org/10.1038/ncomms7377.

Patnaik A, Kang SP, Rasco D, Papadopoulos KP, Elassaiss-Schaap J, Beeram M, et al. Phase I study of pembrolizumab (MK-3475; Anti-PD-1 monoclonal antibody) in patients with advanced solid tumors. Clin Cancer Res. 2015:21(19):4286-93. https://doi.org/10.1158/1078-0432.CCR-14-2607.

Rekhtman N, Paik PK, Arcila ME, Tafe LJ, Oxnard GR, Moreira AL, et al. Clarifying the spectrum of driver oncogene mutations in biomarker-verified squamous carcinoma of lung: lack of EGFR/KRAS and presence of PIK3CA/AKT1 mutations. Clin Cancer Res. 2012;18(4):1167-76. https:// doi.org/10.1158/1078-0432.CCR-11-2109.

Rolfo C, Castiglia M, Hong D, Alessandro R, Mertens I, Baggerman G, et al. Liquid biopsies in lung cancer: the new ambrosia of researchers. Biochem Biophys Acta. 2014;1846(2):539-46. https://doi.org/10.1016/j. bbcan.2014.10.001.

Schwarzenbach H, Hoon DS, Pantel K. Cell-free nucleic acids as biomarkers in cancer patients. Nat Rev Cancer. 2011;1 (6):426-37. https://doi.org/ 10.1038/nrc3066.

Shi X, Duan H, Liu X, Zhou L, Liang Z. Genetic alterations and protein expression in combined small cell lung cancers and small cell lung cancers arising from lung adenocarcinomas after therapy with tyrosine kinase inhibitors. Oncotarget. 2016;7(23):34240-9. https://doi.org/10. 18632/oncotarget.9083.

Skidmore Z, Wagner A, Lesurf R, Campbell K, Kunisaki J, Griffith O, et al. GenVisR: genomic visualizations in R. Bioinformatics. 2016;32:3012-4.

Sobol RE, Astarita RW, Hofeditz C, Masui H, Fairshter R, Royston I, et al. Epidermal growth factor receptor expression in human lung carcinomas defined by a monoclonal antibody. J Natl Cancer Inst. 1987;79(3):403-7.

Spindler KG, Boysen AK, Pallisgard N, Johansen JS, Tabernero J, Sorensen MM, et al. Cell-free DNA in metastatic colorectal cancer: a systematic review and meta-analysis. Oncologist. 2017;22(9):1049-55. https://doi.org/10. 1634/theoncologist.2016-0178. 
Stroun M, Anker P, Maurice P, Lyautey J, Lederrey C, Beljanski M. Neoplastic characteristics of the DNA found in the plasma of cancer patients. Oncology. 1989;46(5):318-22. https://doi.org/10.1159/000226740.

Talevich E, Shain AH, Botton T, Bastian BC. CNVkit: genome-wide copy number detection and visualization from targeted DNA sequencing. PLoS Comput Biol. 2016;12(4):e1004873. https://doi.org/10.1371/journal.pcbi. 1004873.

ThermoFisher Scientific. Oncomine Solid Tumour Kits. https://www.thermofish er.com/se/en/home/clinical/diagnostic-testing/condition-disease-diagn ostics/oncology-diagnostics/oncomine-solid-tumour-kits.html.

Thompson JCYS, Troxel AB, et al. Detection of therapeutically targetable driver and resistance mutations in lung cancer patients by next-generation sequencing of cell-free circulating tumor DNA. Clin Cancer Res. 2016;22(23):5772-82. https://doi.org/10.1158/1078-0432.CCR-16-1231.

Travis WD, Brambilla E, Burke AP, Marx A, Nicholson AG. Tumours of the lung, pleura, thymus and heart. Lyon: IARC Press WHO classification of tumors; 2004.

Tsao AS, Scagliotti GV, Bunn PA Jr, Carbone DP, Warren GW, Bai C, et al. Scientific advances in lung cancer 2015. J Thorac Oncol. 2016;11(5):613-38. https://doi.org/10.1016/j.jtho.2016.03.012.

Vogelstein B, Papadopoulos N, Velculescu VE, Zhou S, Diaz LA Jr, Kinzler KW. Cancer genome landscapes. Science. 2013;339(6127):1546-58. https:// doi.org/10.1126/science.1235122.

Wickham H. ggplot2: elegant graphics for data analysis. New York: Springer; 2016
Wu YL, Lee V, Liam CK, Lu S, Park K, Srimuninnimit V, et al. Clinical utility of a blood-based EGFR mutation test in patients receiving first-line erlotinib therapy in the ENSURE, FASTACT-2, and ASPIRATION studies. Lung Cancer. 2018;126:1-8. https://doi.org/10.1016/j.lungcan.2018.10.004.

Yaung SJ, Krishna S, Xi L, Ju C, Palma JF, Schmid M. Assessment of a highly curated somatic oncology database to aid in the interpretation of clinically important variants in next-generation sequencing results. J Mol Diagn. 2020. https://doi.org/10.1016/j.jmoldx.2020.08.004.

Zhou Q, Li W, Leng B, Zheng W, He Z, Zuo M, et al. Circulating cell free DNA as the diagnostic marker for ovarian cancer: a systematic review and metaanalysis. PLoS ONE. 2016;11(6):e0155495. https://doi.org/10.1371/journal. pone.0155495.

Zojer N, Dekan G, Ackermann J, Fiegl M, Kaufmann H, Drach J, et al. Aneuploidy of chromosome 7 can be detected in invasive lung cancer and associated premalignant lesions of the lung by fluorescence in situ hybridisation. Lung Cancer. 2000;28(3):225-35. https://doi.org/10.1016/ s0169-5002(00)00097-0.

\section{Publisher's Note}

Springer Nature remains neutral with regard to jurisdictional claims in published maps and institutional affiliations.
Ready to submit your research? Choose BMC and benefit from:

- fast, convenient online submission

- thorough peer review by experienced researchers in your field

- rapid publication on acceptance

- support for research data, including large and complex data types

- gold Open Access which fosters wider collaboration and increased citations

- maximum visibility for your research: over $100 \mathrm{M}$ website views per year

At BMC, research is always in progress.

Learn more biomedcentral.com/submissions 